\title{
An Integrated Deployment Planning Tool for Indoor Building Sensor Networks
}

\author{
Rong-Shue Hsiao*, Ding-Bing Lin, Hsin-Piao Lin, \\ Chen-Hua Chung and Fu-Chiang Kang \\ Graduate Institute of Computer and Communication Engineering, \\ National Taipei University of Technology, Taipei 10608, Taiwan
}

(Received December 24, 2013; accepted March 5, 2014)

Key words: $\quad$ wireless sensor networks, ZigBee, sensor deployment, indoor radio channel, building automation systems

Wireless sensor networks have become an increasingly feasible approach for building automation applications. However, radio propagation suffers from largescale path loss, shadowing, multipath fading and other radio interference problems in building environments. Therefore, deployment of sensor nodes in a dynamic in-building environment is a complex operation that requires expert knowledge and experience. In this paper, we present a deployment planning tool that can provide a set of useful tools for deploying a wireless sensor network in a building environment. The proposed deployment planning tool provides design tools that can assist users in developing a network infrastructure to support reliable communication. To evaluate the effectiveness of this tool, we designed a sensor network using this tool and deployed a wireless sensor network in our testbed. The experimental results showed that the network performance achieved a high level of reliability.

\section{Introduction}

In recent years, the technology of wireless sensor networks (WSNs) has been attracting extensive research and development efforts to replace the traditional wired solutions for building automation systems (BAS). ZigBee-based WSNs are based on the IEEE 802.15.4 physical (PHY) and media access control (MAC) layers to provide standards-based, reliable wireless data transfer. ${ }^{(1)}$ However, radio propagation suffers from large-scale path loss, shadowing, multipath fading and other radio interference problems in building environments. Therefore, the reliability of wireless network communication is the main concern in indoor environments. ${ }^{(2)}$ Overcoming the reliability problem of wireless network communications is crucial for BAS applications.

"Corresponding author: e-mail: rshsiao@ntut.edu.tw 
WSNs are considered as autonomous ad hoc networks that are able to organize themselves without a predefined infrastructure. However, it is necessary to have a networking infrastructure to solve the problems of communication reliability, energy constraints, and so forth. ${ }^{(3,4)}$ Cerpa and Estrin ${ }^{(5)}$ proposed a topology that acts as a communication backbone to save energy. Alvarez et al. ${ }^{(6)}$ established a virtual network with the topology of a mesh of stars, which also established a reliable and efficient high-level communication system. To support the design of wireless infrastructure for building applications, Guinard et al.(7) proposed a design framework that consists of a number of integrated model-driven tools to support each phase of the design, from requirements to operation. They argued that the topology and positioning of sensor nodes are the essential considerations at the deployment planning stage to ensure a reliable communication infrastructure.

In this paper, we propose a deployment planning tool based on a hierarchical clustering topology that is used to support the deployment of a WSN for building automation. The procedure of deployment planning includes requirements, design and network evaluation phases. The requirements phase defines the system requirements of the building environment. The design phase consists of sensor-cluster design and backbone design. The final phase is the evaluation of the network quality.

\section{System Architecture}

The system consists of design and evaluation tools that are developed by integrating the ns-2 network simulator. However, ns-2 does not support simulation of radio propagation under obstructed conditions. Therefore, we extended its source code to simulate the propagation loss when there is an obstacle.

\subsection{Requirements}

Network topology: In the system, we define the network topology and indoor radio propagation model. The network topology and physical location of sensor nodes strongly affect the performance of the network from the perspective of reliable communication. Node clustering techniques are often used in the context of WSNs, and as these networks are composed of a very large number of nodes, a hierarchical organization of the network units might also prove to be extremely useful. In addition, node clustering can support data fusion and reduce the probability of packet collision. ${ }^{(8)}$ The network infrastructure of a hierarchical cluster-based network topology is shown in Fig. 1. A sensor node in a cluster sends/receives data to/from the cluster head; the cluster head communicates with the router and can also be used as a router, the router communicates with the gateway, and the gateway communicates with the sink, the gateway being the same as the sink in a one-floor system. The primary objective of node clustering was to attempt to reduce the amount of data delivered to the sink, improving the communication performance.

Indoor radio channel: The quality of radio communication in the indoor environment depends heavily on the characteristics of the indoor radio channel. The indoor radio channel depends on factors that include building structure, layout of rooms, and the type of construction materials used. In the indoor environment, the log-normal 


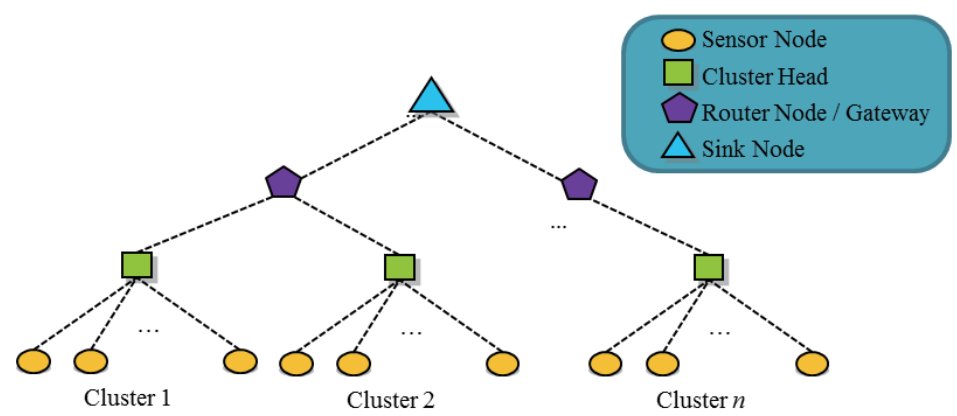

Fig. 1. (Color online) Network infrastructure of hierarchical clustering.

shadowing propagation model is very suitable to be used. In addition, we also have considered floor and partition loss in this system. ${ }^{(9)}$

Medium access control: The MAC protocol is responsible for providing reliable communications between a node and its immediate neighbors, which helps to avoid collisions and improve efficiency. As ZigBee is based on the IEEE 802.15.4 protocol stack, the MAC protocol used by the standard is the CSMA/CA (Carrier Sense Multiple Access with Collision Avoidance) protocol. However, the CSMA-based MAC protocol may suffer from drastic network performance degradation with the number of neighbor nodes owing to the increasing number of message collisions. This performance degradation is even more acute owing to the impact of the hidden-node problem, which is caused by hidden-node collisions. ${ }^{(10)}$ The transmission range depends on the transmission power. The carrier sensing range is typically larger than the transmission range, for instance, two times larger than the transmission range. ${ }^{(11,12)}$

\subsection{Design}

In the design phase, the system supports a link quality estimation tool for the design of the sensor cluster and backbone. For the simulation, a pair of nodes (transmitter and receiver) was set up. Using this tool, there are some parameters that need to be set before placing sensor nodes at the desired locations. The related parameters include the path loss exponent $n$ and the zero-mean Gaussian random variable with standard deviation $\sigma$ of the log-normal shadowing propagation model. In the building environment, the path loss exponent $n$ can be set to calculate the partition loss. If the user does not input the parameters, a default value of 2 (for free space) will be set for the path loss exponent $n$, and the standard deviation $\sigma$ will be 4 . In addition, the device-related parameters have to be configured, such as the transmit power of the sensor nodes and the threshold of the received power. The threshold value is used to determine whether packets can be received completely.

In an indoor building environment, the link quality can be categorized into three regions: (i) connected region (high packet reception rate, larger than 99\%), (ii) transitional region (data reception rate is variable), and (iii) disconnected region (very 
low data reception rate).(3) The range of each region depends on the transmit power and the operational environment. An example of link quality estimation is shown in Fig. 2 , in which the distance of the transmitter and receiver is set to $8 \mathrm{~m}$. Then, the packet reception rate is $100 \%$. To ensure reliable communication, the separation of two nodes must be kept inside the connected range.

Furthermore, channel quality is critical in the sensor node cluster. In the area of a high-density sensor node cluster, the impact of channel contention must be considered. This tool also supports the simulation of channel contention. This function can help the user decide how many sensor nodes should be deployed in a sensor cluster. We configured a sensor cluster that includes a cluster head with different numbers of sensor nodes. Each sensor node remains inside the connected range with the cluster head. As shown in Fig. 3, the simulation results show that channel contention occurs. When the number of sensor nodes is increased to 4 , the packet reception rate (PRR) decreases to $74.36 \%$. Therefore, the number of sensor nodes must be limited to avoid heavy packet loss and retransmission. Also, the backbone (router) deployment should consider the number of neighboring nodes, and if the nodes belong to the same carrier sensing set, the nodes may transmit simultaneously.

By using the supporting tools of the design phase, the locations of each sensor node and router nodes can be decided on the basis of the predefined network topology. The overall network is then formed.

\begin{tabular}{|c|c|c|c|c|c|c|}
\hline 吅 & & & \multicolumn{3}{|c|}{ Link Quality Estimation } & $-\square \times$ \\
\hline Model Parameters & & & \multicolumn{2}{|c|}{ Device Parameters } & \multirow{3}{*}{$\begin{array}{l}\mathrm{dBm} \\
\mathrm{m}\end{array}$} & Start \\
\hline Path Loss Exponent & 2 & & Transmit Power & -8 & & \multirow{2}{*}{ Packet Reception Rate } \\
\hline Standard Deviation & 4 & & T-R Distance & 8 & & \\
\hline Close-in Reference Distance & 1 & $\mathrm{~m}$ & Packet Size & 84 & Bytes & 100 \\
\hline
\end{tabular}

Fig. 2. (Color online) An example of link quality estimation.

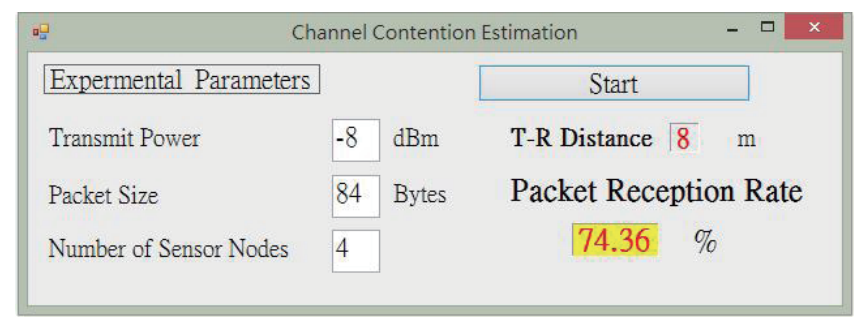

Fig. 3. (Color online) An example of channel quality estimation. 


\subsection{Network quality evaluation}

The most important design issue of a WSN application is to reliably deliver the detected data from the sensor node to the sink. In this phase, the network quality is then evaluated by measuring the packet reception rate at the sink node.

\section{Experimental Results and Evaluation}

To evaluate the effectiveness of our deployment planning tool, we used the tool to design a WSN deployment plan on two different building environments. First, we deployed the sensor nodes (end devices) in the sensing area to form a number of sensor clusters. Then, the router nodes and coordinator/sink were deployed to form the backbone of the network. According to the deployment plan, we used the XBee series $2 \mathrm{RF}$ module as our sensor node to deploy the network. All the transmit powers of the nodes were set at the minimum level $(-8 \mathrm{dBm})$.

We evaluated the network quality in three parts, which are the three different event sources (sensor cluster) connecting to the coordinator (sink). One is that the signal from a sensor node passes through a long portion of free space (line-of-sight, LOS) to the coordinator. The second is a signal from a sensor cluster to the coordinator. The third is that the signal passes through obstacles (nonline-of-sight, NLOS), such as glass and concrete walls. The transmission rates were set to 1, 2, and 3 packets/s, respectively. Data were passed from three event sources individually. The PRR was measured at the sink node. The experimental and simulation results are shown in Table 1.

The PRR was lower in the second area than in the other areas because channel contention occurred. Also, most of the PRR values were better in the experimental results than in the simulation results. We think that this is probably caused by the differences in environmental parameters between the simulation and empirical measurement; another possibility is that it is caused by some reliable communication mechanisms that are provided by the sensor node module.

Table 1

(a) Experimental results.

\begin{tabular}{lccc}
\hline \multirow{2}{*}{ Transmission rate } & \multicolumn{3}{c}{ PRR (Building 1/Building 2) } \\
\cline { 2 - 4 } & No. 1 & No. 2 & No. 3 \\
\hline 1 packet/s & $97.1 \% / 99.5 \%$ & $88.1 \% / 99.3 \%$ & $97.9 \% / 99.9 \%$ \\
2 packets/s & $94.0 \% / 99.1 \%$ & $86.5 \% / 98.4 \%$ & $95.7 \% / 99.6 \%$ \\
3 packets/s & $93.8 \% / 98.3 \%$ & $86.3 \% / 96.5 \%$ & $92.5 \% / 99.2 \%$ \\
\hline
\end{tabular}

(b) Simulation results.

\begin{tabular}{lccc}
\hline \multirow{2}{*}{ Transmission rate } & \multicolumn{3}{c}{ PRR } \\
\cline { 2 - 4 } & No. 1 & No. 2 & No. 3 \\
\hline 1 packet/s & $95.9 \%$ & $85.71 \%$ & $99.51 \%$ \\
2 packets/s & $96.9 \%$ & $83.37 \%$ & $99.37 \%$ \\
3 packets/s & $95.0 \%$ & $80.62 \%$ & $98.3 \%$ \\
\hline
\end{tabular}




\section{Conclusions}

Reliable communication is a critical issue in BAS applications. Developing the infrastructure of a wireless sensor network is essential for solving the unreliable communication problem. In this paper, we have proposed a deployment planning tool to assist users in developing a WSN infrastructure of hierarchical clustering. The tool development considered both the characteristics of indoor radio communication and wireless medium access, and was shown to be effective for reliable communication in building environments. In the future, we will further develop network-routing-related functions to assist sensor node deployment.

\section{References}

1 ZigBee Alliance: ZigBee Specification Document 053474r17 (2007).

2 B. Krishnamachari: Networking Wireless Sensors (Cambridge University Press, New York, 2005) Chap. 5.

3 K. Beydoun and V. Felea: Proc. Int. Symp. on Collaborative Technologies and Systems, 2008, pp. 58-65.

4 A. Guinard, A. McGibney and D. Pesch: Proc. First ACM Workshop on Embedded Sensing Systems for Energy-Efficiency in Buildings, 2009, pp. 25-30.

5 A. Cerpa and D. Estrin: IEEE Trans. Mob. Comput. 3 (2004) 272.

6 C. Alvarez, J. Diaz, J. Petit, J. Rolim and M. Serna: The 3rd Int. Mobility and Wireless Access Workshop, 2004, pp. 106-110.

7 A. Guinard, M. S. Aslam, D. Pusceddu, S. Rea, A. McGibney and D. Pesch: IEEE 36th Conf. on Local Computer Networks, 2011, pp. 649-656.

8 O. Younis, S. Fahmy and P. Santi: Int. J. Distrib. Sens. Netw. 1 (2005) 305.

9 T. S. Rappaport: Wireless Communications Principles and Practice (Prentice Hall PTR, New Jersey, 2002) 2nd ed., Chap. 4.

10 A. Koubâa, R. Severino, M. Alves and E. Tovar: IEEE Trans. Ind. Inf. 5 (2009) 299.

11 V. S. Arulmurugan and S. Vijayan: Aust. J. Basic \& Appl. Sci. 6 (2012) 215.

12 Y. Yang and R. Kravets: IEEE Trans. Mob. Comput. 4 (2005) 363. 